\title{
Nanomechanics of Carbon Tubes: Instabilities beyond Linear Response
}

\author{
B. I. Yakobson,* C. J. Brabec, and J. Bernholc \\ Department of Physics, North Carolina State University, Raleigh, North Carolina 27695
}

(Received 22 September 1995)

\begin{abstract}
Carbon nanotubes subject to large deformations reversibly switch into different morphological patterns. Each shape change corresponds to an abrupt release of energy and a singularity in the stressstrain curve. These transformations, simulated using a realistic many-body potential, are explained by a continuum shell model. With properly chosen parameters, the model provides a remarkably accurate "roadmap" of nanotube behavior beyond Hooke's law.
\end{abstract}

PACS numbers: 61.46.+w, 02.70.Ns, 46.30.Lx, 85.40.Ux

It took the Daedalus' "hollow molecules" [1] two decades to become incarnated in a family of fullerenes [2,3], remaining since then an object of keen interest: Their growth, atomic makeup, electronic and mechanical properties, doping, and intercalation are all being investigated. The variety of shapes is usually associated with embedded pentagons or heptagons, which accommodate a positive or a negative Gaussian curvature of otherwise hexagonal graphene scrolls. Small elastic deviations from cylindrical shape have also been observed [4]. Latest findings in transmission electron microscopy of nanotubes [5-7] suggest that even largely distorted configurations can be due to elastic deformations with no atomic defects involved. Such a suggestion is provocative for a graphitelike material, commonly perceived as rather brittle [8], and poses important questions: What range of strain can nanotubes sustain elastically, and what kind of patterns can they exhibit under different mechanical load? It is closely related with the ultimate strength of nanotubes and their lateral stiffness when used as quantum wires or proximal probes. While direct experimental answers are pending, a better control of the forces involved, a sentient atomistic modeling can already provide useful guidelines.

Recent development of many-body interatomic potentials $[9,10]$ made possible realistic molecular dynamics (MD) simulations of carbon-made systems. We carried out such studies for nanotubes under generic modes of mechanical load: axial compression, bending, and torsion. A remarkable synergism between the methods of MD and those of macroscopic structural mechanics was found. A singular behavior of the nanotube energy at certain levels of strain corresponds to abrupt changes in morphology, when a switch between distinct patterns occurs. These transformations can be explained with a continuous tubule model. With properly chosen parameters, this analytical model predicts nanotube behavior not only at small deformations but also beyond the linear response.

One of the outstanding features of fullerenes is their hollow structure; they consist of atoms densely packed along a closed surface that defines the overall shape. This should also manifest itself in dynamic properties of molecules, resembling so much the macroscopic objects known in continuum elasticity as shells $[11,12]$. Macroscopic shells and rods have long been of interest: The first study dates back to Euler, who discovered the elastic instability. A rod subject to longitudinal compression remains straight but shortens by some fraction $\varepsilon$, proportional to the force, until a critical value (Euler force) is reached. It then becomes unstable and buckles sideways at $\varepsilon>\varepsilon_{\mathrm{cr}}$, while the force almost does not vary [13]. For hollow tubules there is also a possibility of local buckling, in addition to buckling as a whole. Therefore, more than one bifurcation can be expected.

In application to fullerenes, the theory of shells may serve as a useful guide, but its relevance for a covalentbonded system of only a few atoms in diameter is far from obvious. MD simulations are much better suited for objects that small [14]. The discussion below proceeds therefore in two, almost parallel, paths. First, the results of MD simulations are presented for a nanotube under axial compression. We then briefly introduce concepts of elasticity of shells and show how to adapt them to nanotubes. MD results for other modes of load follow and are compared with those suggested by the continuum model.

Figure 1 shows a simulated nanotube exposed to $a x$ ial compression. The atomic interaction was modeled by the Tersoff-Brenner potential, which reproduces the lattice constants, binding energies, and the elastic constants of graphite and diamond [10]. The end atoms were shifted along the axis by small steps, and the whole tube was relaxed by a conjugate-gradient method while keeping the ends constrained. At small strains the total energy [Fig. 1(a)] grows as $E(\varepsilon)=\frac{1}{2} E^{\prime \prime} \varepsilon^{2}$, where $E^{\prime \prime}=59 \mathrm{eV} /$ atom. The presence of four singularities at higher strains is quite a striking feature, and the patterns 1(b)-1(e) illustrate the corresponding morphological changes. The shading indicates strain energy per atom, equally spaced from below $0.5 \mathrm{eV}$ (brightest) to above $1.5 \mathrm{eV}$ (darkest). The sequence of singularities in $E(\varepsilon)$ corresponds to a loss of molecular symmetry from $D_{\infty h}$ to $S_{4}, D_{2 h}, C_{2 h}$, and $C_{1}$. Can this evolution be put in the framework of continuum elasticity?

The intrinsic symmetry of a graphite sheet is hexagonal. Since the elastic properties of a two-dimensional 


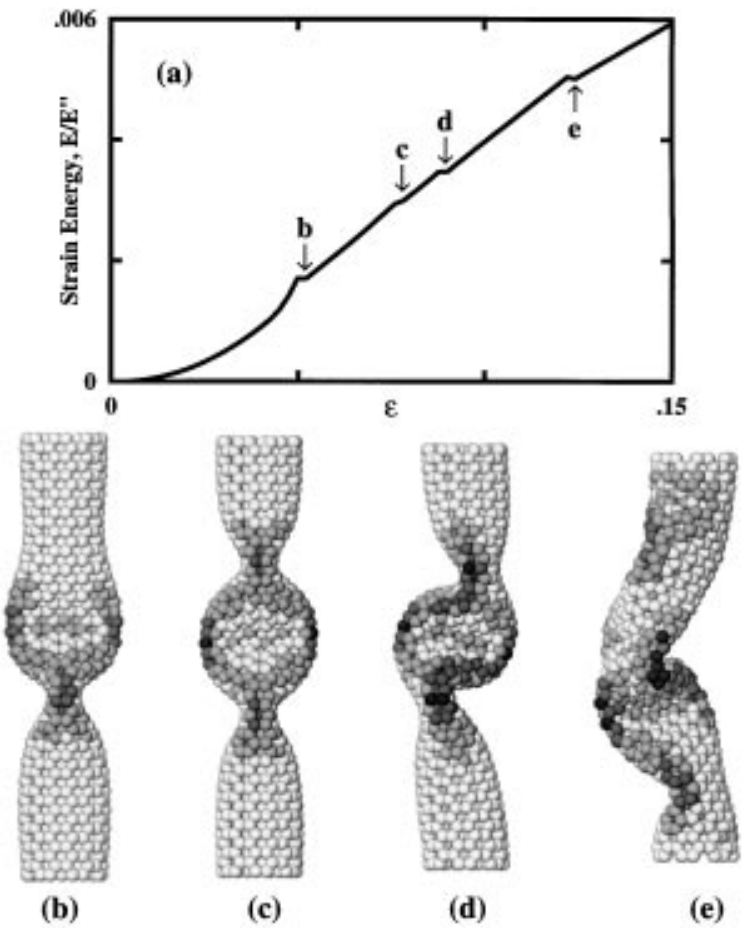

FIG. 1. MD-simulated nanotube of length $L=6 \mathrm{~nm}$, diameter $d=1 \mathrm{~nm}$, and armchair helicity $(7,7)$ under axial compression. The strain energy (a) displays four singularities corresponding to shape changes. At $\varepsilon_{1}=0.05$ the cylinder buckles into the pattern (b), displaying two identical flattenings - "fins" perpendicular to each other. Further increase of $\varepsilon$ enhances this pattern gradually until at $\varepsilon_{2}=0.076$ the tube switches to a three-fin pattern (c), which still possesses a straight axis. In a buckling sideways at $\varepsilon_{3}=0.09$ the flattenings serve as hinges, and only a plane of symmetry is preserved (d). At $\varepsilon_{4}=0.13$ an entirely squashed asymmetric configuration forms (e) (see text).

hexagonal structure are isotropic $[11,15]$, it can be approximated by a uniform shell with only two elastic parameters: flexural rigidity $D$, and its resistance to an in-plane stretching, the in-plane stiffness $C$. The energy of a shell is given by a surface integral of the quadratic form of local deformation $[11,12]$,

$$
\begin{array}{r}
E=\frac{1}{2} \iint\left\{D\left[\left(\kappa_{x}+\kappa_{y}\right)^{2}-2(1-\nu)\left(\kappa_{x} \kappa_{y}-\kappa_{x y}^{2}\right)\right]\right. \\
+\frac{C}{\left(1-\nu^{2}\right)}\left[\left(\varepsilon_{x}+\varepsilon_{y}\right)^{2}-2(1-\nu)\right. \\
\left.\left.\times\left(\varepsilon_{x} \varepsilon_{y}-\varepsilon_{x y}^{2}\right)\right]\right\} d S
\end{array}
$$

where $\kappa$ is the curvature variation, $\varepsilon$ is the in-plane strain, and $x$ and $y$ are local coordinates (see Fig. 2, inset). In order to adapt this formalism to a graphitic tubule, the values of $D$ and $C$ can be identified by comparison with the detailed $a b$ initio and semiempirical studies of nanotube energetics at small strains [16,17]. Indeed, the second derivative of total energy with respect to axial strain corresponds to the in-plane rigidity $C$. Similarly, the strain energy as a function of tube diameter

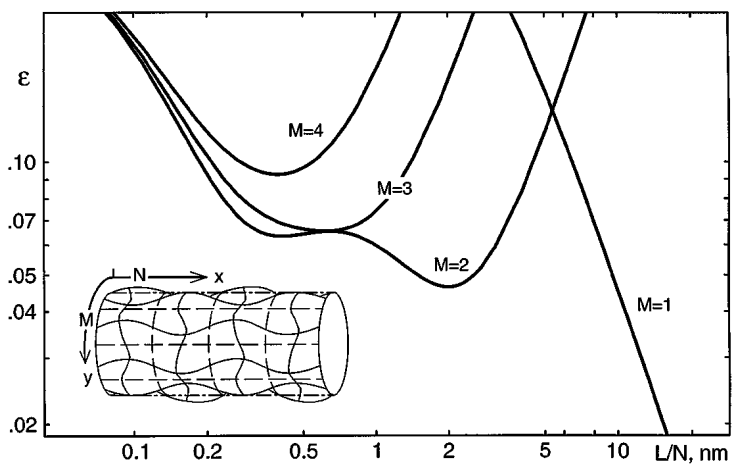

FIG. 2. The critical strain levels for a continuous, $1 \mathrm{~nm}$ wide tube as a function of its scaled length $L / N$. A buckling pattern $(M, N)$ is defined by the number of half waves $2 M$ and $N$ in the $y$ and $x$ directions, respectively, e.g., a $(4,4)$ pattern is shown in the inset.

$d$ corresponds to $2 D / d^{2}$ in Eq. (1). Using the data of Ref. [17], we obtain $C=59 \mathrm{eV} /$ atom $=360 \mathrm{~J} / \mathrm{m}^{2}$ and $D=0.85 \mathrm{eV}$. The Poisson ratio $\nu=0.19$ was extracted from a reduction of the diameter of a tube stretched in simulations. The same value is obtained from experimental elastic constants of single crystal graphite [8]. One can make a further step towards a more tangible picture of a tube as having wall thickness $h$ and Young modulus $Y$. Using the standard relations $D=Y h^{3} / 12\left(1-\nu^{2}\right)$ and $C=Y h$ [11], one finds $Y=$ $5.5 \mathrm{TPa}$ and $h=0.066 \mathrm{~nm}$ [18]. With these parameters, linear stability analysis [11,12] allows us to assess the nanotube behavior under strain.

Consider briefly the case of imposed axial strain. A trial perturbation of a cylinder has a form of Fourier harmonics, with $M$ azimuthal lobes and $N$ half waves along the tube (Fig. 2, inset), i.e., sines and cosines of arguments $2 M y / d$ and $N \pi x / L$. At a critical level of the imposed strain, $\varepsilon_{c}(M, N)$, the energy variation (1) vanishes for this shape disturbance. The cylinder becomes unstable and lowers its energy by assuming an $(M, N)$ pattern. For tubes of $d=1 \mathrm{~nm}$ with the shell parameters identified above, the critical strain is shown in Fig. 2. According to these plots, for a tube with $L>10 \mathrm{~nm}$ the bifurcation is first attained for $M=1, N=1$. The tube preserves its circular cross section and buckles sideways as a whole; the critical strain is close to that for a simple rod,

$$
\varepsilon_{c}=\frac{1}{2}(\pi d / L)^{2},
$$

or 4 times less for a tube with hinged edges [11]. For a shorter tube the situation is different. The lowest critical strain occurs for $M=2$ (and $N \geq 1$, see Fig. 2), with a few separated flattenings in directions perpendicular to each other, while the axis remains straight. For such a local buckling, in contrast to (2), the critical strain depends little on length and estimates to $\varepsilon_{c}=$ $4 \sqrt{D / C} d^{-1}=(2 / \sqrt{3})\left(1-\nu^{2}\right)^{-1 / 2} h d^{-1}$ in the Lorenz limit [19]. For a nanotube one finds

$$
\varepsilon_{c}=(0.077 \mathrm{~nm}) d^{-1} \text {. }
$$


Specifically, for the $1 \mathrm{~nm}$ wide tube of length $L=6 \mathrm{~nm}$, the lowest critical strains occur for the $M=2$ and $N=$ 2 or 3 (Fig. 2), and are close to the value obtained in MD simulations [Fig. 1(a)]. This is in accord with the twoand three-fin patterns seen in Figs. 1(b) and 1(c). Higher singularities cannot be quantified by the linear analysis, but they look like a sideways beam buckling, which at this stage becomes a nonuniform object.

Axially compressed tubes of greater length and/or tubes simulated with hinged ends (equivalent to a doubled length [11]) first buckle sideways as a whole at a strain consistent with (2). After that, the compression at the ends results in bending and a local buckling inward. This illustrates the importance of the "beam-bending" mode, the softest for a long molecule and most likely to attain significant amplitudes due to either thermal vibrations [20] or environmental forces. In simulations of bending, a torque rather than force is applied at the ends, and the bending angle $\theta$ increases stepwise. While a notch in the energy plot [Fig. 3(a)] can be mistaken for numerical noise, its derivative $d E / d \theta$ unambiguously shows an increase in tube compliance-a signature of a buckling event [12]. In bending, only one side of a tube is compressed and thus can buckle. Assuming that it buckles when its local strain $\varepsilon=K d / 2$, where $K$ is the local curvature, is close to that in axial compression [Eq. (3)], we estimate the critical curvature as

$$
K_{c}=(0.155 \mathrm{~nm}) d^{-2} .
$$

This is in excellent agreement (within 4\%) with extensive simulations of single wall tubes of various diameters, helicities, and lengths [6]. Due to end effects, the average curvature is less than the local one, and the simulated segment buckles somewhat earlier than at $\theta_{c}=K_{c} L$, which is accurate for longer tubes.

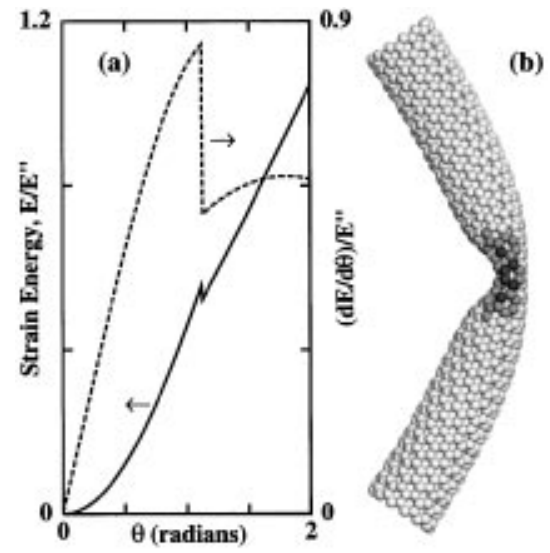

FIG. 3. Bending of a tubule of $(13,0)$ helicity, $8 \mathrm{~nm}$ long and $1 \mathrm{~nm}$ wide. (a) The strain energy curve (normalized to its second derivative) switches from harmonic to linear at the buckling point, while the force (dashed line) drops and remains almost constant afterwards. (b) Beyond the buckling point, a distinct kink shape develops.
In simulation of torsion, the increase of azimuthal angle $\phi$ between the tube ends results in the changes of energy and morphology shown in Fig. 4. In the continuum model, the analysis based on Eq. (1) is similar to that outlined above, except that it involves skew harmonics of arguments like $N \pi x / L \pm 2 M y / d$ [12]. For overall beam buckling $(M=1)$,

$$
\phi_{c}=2(1+\nu) \pi,
$$

and for the cylinder-helix flattening $(M=2)$,

$$
\phi_{c}=\left(0.055 \mathrm{~nm}^{3 / 2}\right) L d^{-5 / 2} \text {. }
$$

The latter should occur first for $L \leq 136 d^{5 / 2} \mathrm{~nm}$, which is true for all the tubes we simulated. However, in simulations it occurs later than predicted by Eq. (6). The ends, kept circular in simulation, which is physically justifiable by a presence of rigid caps on normally closed ends of a molecule, deter the through flattening necessary for the helix to form (unlike the local flattening in the case of an axial load).

Simulations of nanotubes under mechanical duress lead to shapes very different in appearance. At the same time there are robust traits in common: A deformation, proportional to the force within Hooke's law, eventually leads to a collapse of the cylinder and an abrupt change in pattern, or a sequence of such events. The presence of a snap-through buckling of nanotubes allows for a possibility of "shape memory" when, in an unloading cycle, the switch between patterns occurs at a somewhat lower level of strain. A small hysteresis observed in simulations is practically eliminated by thermal motion and can barely be caught in experiment. More interestingly, video imaging of the simulations at even a low temperature $(50 \mathrm{~K})$ shows strongly enhanced thermal vibrations in the vicinity of every pattern switch, while before and after the transition only barely noticeable ripples are seen.

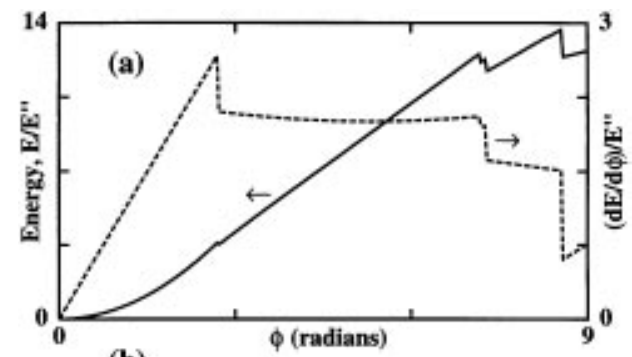

(b)

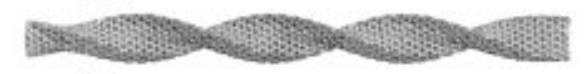

(c)

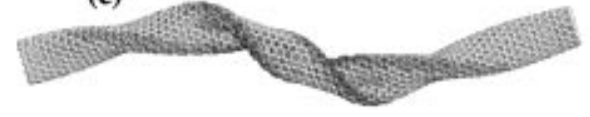

FIG. 4. The energy of a sawtooth $(13,0)$ tube, $23 \mathrm{~nm}$ long and $1 \mathrm{~nm}$ wide, as a function of torsion angle $\phi$ (a). At $\phi_{1}=2.7$ the cylinder flattens into a straight axis spiral (b). At $\phi_{2}=7.2$ the whole helix buckles sideways with a loss of the straight axis (c). It coils up in a "forced" tertiary structure at $\phi_{3}=8.6$. 
Physically, this indicates softening of the system, when one of the eigenvalues tends to zero in the vicinity of the bifurcation.

Despite the very large local strain in the nanotubes, we did not see any bond breaking or switching. Overall, the simulations show that graphitic layers remain highly elastic. Conversely, this conclusion, consistent with the recent detection of the patterns [5-7], serves as another verification of the potential $[9,10]$ used, which thus proves to be realistic at large strain. This abets the study of axial tension, where no shape transformations occur up to an extreme dilation. Preliminary MD results (at $T=50 \mathrm{~K}$ ) show a tube stretched by almost $40 \%$ with no damage to its graphitic arrangement. Only at this point an atomic disorder is seen to nucleate suddenly, the tube weakens, and, after a pulled out long chain of atoms breaks, separates into two fragments [21]. This stretchy, rather than brittle, behavior for such a hard "material" indicates extreme strength; it deserves further study, thorough in atomic order analysis and possibly in refinements of the potential.

An atomistic modeling of multilayer tubes remains expensive. It makes extrapolation of the continuum model tempting, but involves an interlayer van der Waals interaction. The flexural rigidity scales as $\sim h^{3}$ in the case of a coherent, and as $\sim h$ for an incoherent stack of layers [22], sliding with respect to each other when the tube is deformed; this affects the mechanical properties and still has to be investigated.

We did not simulate tubes under hydrostatic pressure. At this scale, anisotropic lateral forces in a molecular crystal packing are more plausible than a uniform pressure [23]. Instead, one can resort to continuum elasticity and estimate a pressure leading to an inward buckling as $p_{c} \approx 2 Y(h / d)^{3}$ [12], that is, thousands of atmospheres for a nanometer tube. However, it drops fast with the diameter and is assisted by a flattening effect of twisting or bending [24] and by van der Waals attraction between the opposite walls. Elastic collapse can thus impede the growth of single shell tubes with $d>3 \mathrm{~nm}$.

In summary, our simulations show that carbon nanotubes are remarkably resilient, sustaining extreme strain with no signs of brittleness, plasticity, or atomic rearrangements. Their peculiar behavior beyond Hooke's law can be well described by a continuum model: At large deformations an abrupt release of energy is accompanied by a reversible switch into a different morphological pattern. With properly chosen tubule parameters $(\nu \approx 0.19$, $Y \approx 5.5 \mathrm{TPa}, h \approx 0.66 \AA$ ), the critical deformations at which these buckling events occur can be estimated by Eqs. (2)-(6). Corroborated by recent experimental observations [5-7], this model provides a useful tool for fullerene studies.

*Electronic address: yakobson@ncsu.edu
[1] D. Jones, New Sci. 32, 245 (1966); D. Jones, The Inventions of Daedalus (Freeman, Oxford, 1982).

[2] H. Kroto, J. Heath, S. O'Brien, R. Curl, and R. Smalley, Nature (London) 318, 162 (1985); W. Krätschmer, L. Lamb, K. Fostiropoulos, and D. Huffman, Nature (London) 347, 354 (1990); S. Iijima, Nature (London) 354, 56 (1991) .

[3] Special issue edited by H. Kroto, C.N.R. Rao, and E. Osawa, Mater. Res. Soc. Bull. 19, No. 11 (1994).

[4] R. Ruoff et al., Nature (London) 364, 514 (1993).

[5] J. Despres, E. Daguerre, and K. Lafdi, Carbon 33, 87 (1995).

[6] S. Iijima, C. J. Brabec, A. Maiti, and J. Bernholc, J. Chem. Phys. 104, (1996).

[7] N. Chopra, L. Benedict, V. Crespi, M. Cohen, S. Louie, and A. Zettl, Nature (London) 377, 135 (1995); R. Ruoff and D. Lorents, Bull. Am. Phys. Soc. 40, 173 (1995).

[8] B. Kelly, Physics of Graphite (Applied Science, London, 1981); M. Dresselhaus, G. Dresselhaus, K. Sugihara, I. Spain, and H. Goldberg, Graphite Fibers and Filaments (Springer, Berlin, 1988).

[9] J. Tersoff, Phys. Rev. B 37, 6991 (1988).

[10] D. W. Brenner, Phys. Rev. B 42, 9458 (1990).

[11] L. D. Landau and E. M. Lifshitz, Elasticity Theory (Pergamon, Oxford, 1986).

[12] H. Allen and P. Bulson, Background to Buckling (McGraw-Hill, London, 1980); S. Timoshenko and J. Gere, Theory of Elastic Stability (McGraw-Hill, New York, 1988).

[13] R. Feynman, R. Leyton, and M. Sands, The Feynman Lectures in Physics (Addison-Wesley, Reading, 1964), Vol. 2.

[14] U. Landman et al., Science 248, 454 (1990).

[15] This explains why the detailed calculations [16,17] did not reveal significant effects of helicity on elastic properties; it also excludes the "mechanical chirality," a different resistance to left and right twists due to intrinsic helical structure.

[16] G. Adams et al., Science 256, 1792 (1992); A. Lucas, P. Lambin, and R. Smalley, J. Phys. Chem. Solids 54, 587 (1993).

[17] D. Robertson, D. Brenner, and J. Mintmire, Phys. Rev. B 45, 12592 (1992).

[18] $h$ appears to be less than the atomic radius, due to the "open-work" structure of graphite manifesting itself on this scale.

[19] R. Lorenz, Phys. Z 12, 241 (1911).

[20] G. Overney, W. Zhong, and D. Tomanek, Z. Phys. D 27, 93 (1993).

[21] Recent experiments indicate that a monoatomic chain can also be pulled out of a nanotube by a strong electric field; see A. Rinzler, J. Hafner, P. Nikolaev, L. Lou, S. Kim, D. Tomanek, p. Norlander, D. Colbert, and R. Smalley, Science 269, 1550 (1995).

[22] S. Safran, Statistical Thermodynamics of Surface, Interfaces and Membranes (Addison-Wesley, Reading, 1994); D. Srolovitz et al., Phys. Rev. Lett. 74, 1779 (1995).

[23] J. Tersoff and R. Ruoff, Phys. Rev. Lett. 73, 676 (1994).

[24] A. Palmer and J. Martin, Nature (London) 254, 46 (1975). 